\title{
Semantics of MML Query ${ }^{1}$
}

\author{
Grzegorz Bancerek \\ Białystok Technical University \\ Poland
}

Summary. In the paper the semantics of MML Query queries is given. The formalization is done according to [4].

MML identifier: MMLQUERY, version: $\underline{7.12 .02 \quad 4.181 .1147}$

The notation and terminology used here have been introduced in the following papers: [1], [5], [11], [8], [10], [6], [2], [3], [15], [13], [14], [9], [12], and [7].

\section{Elementary Queries}

Let $X$ be a set. A list of $X$ is a subset of $X$. An operation of $X$ is a binary relation on $X$.

Let $x, y, R$ be sets. The predicate $x, y \in R$ is defined by:

(Def. 1) $\langle x, y\rangle \in R$.

Let $x, y, R$ be sets. We introduce $x, y \notin R$ as an antonym of $x, y \in R$.

For simplicity, we use the following convention: $X, Y, z, s$ denote sets, $L, L_{1}$, $L_{2}, A$ denote lists of $X, x$ denotes an element of $X, O, O_{2}, O_{3}$ denote operations of $X$, and $m$ denotes a natural number.

The following proposition is true

(1) For all binary relations $R_{1}, R_{2}$ holds $R_{1} \subseteq R_{2}$ iff for every $z$ holds $R_{1}{ }^{\circ} z \subseteq R_{2}^{\circ} z$.

Let us consider $X, O, x$. We introduce $x O$ as a synonym of $O^{\circ} x$.

Let us consider $X, O, x$. Then $x O$ is a list of $X$.

One can prove the following proposition

\footnotetext{
${ }^{1}$ This work has been supported by the Polish Ministry of Science and Higher Education project "Managing a Large Repository of Computer-verified Mathematical Knowledge" (N N519 385136).

(C) 2012 University of Białystok CC-BY-SA License ver. 3.0 or later ISSN 1426-2630(p), 1898-9934(e)
} 
(2) $x, y \in O$ iff $y \in x O$.

Let us consider $X, O, L$. We introduce $L \mid O$ as a synonym of $O^{\circ} L$.

Let us consider $X, O, L$. Then $L \mid O$ is a list of $X$ and it can be characterized by the condition:

(Def. 2) $L \mid O=\bigcup\{x O: x \in L\}$.

The functor $L \& O$ yielding a list of $X$ is defined as follows:

(Def. 3) $L \& O=\bigcap\{x O: x \in L\}$.

The functor $L$ where $O$ yielding a list of $X$ is defined as follows:

(Def. 4) $L$ where $O=\left\{x: \bigvee_{y}(x, y \in O \wedge x \in L)\right\}$.

Let $O_{2}$ be an operation of $X$. The functor $L$ where $O=O_{2}$ yielding a list of $X$ is defined as follows:

(Def. 5) $L$ where $O=O_{2}=\left\{x: \overline{\overline{x O}}=\overline{\overline{x O_{2}}} \wedge x \in L\right\}$.

The functor $L$ where $O \leq O_{2}$ yielding a list of $X$ is defined by:

(Def. 6) $L$ where $O \leq O_{2}=\left\{x: \overline{\overline{x O}} \subseteq \overline{\overline{x O_{2}}} \wedge x \in L\right\}$.

The functor $L$ where $O \geq O_{2}$ yields a list of $X$ and is defined by:

(Def. 7) $L$ where $O \geq O_{2}=\left\{x: \overline{\overline{x O_{2}}} \subseteq \overline{\overline{x O}} \wedge x \in L\right\}$.

The functor $L$ where $O<O_{2}$ yielding a list of $X$ is defined as follows:

(Def. 8) $L$ where $O<O_{2}=\left\{x: \overline{\overline{x O}} \in \overline{\overline{x O_{2}}} \wedge x \in L\right\}$.

The functor $L$ where $O>O_{2}$ yields a list of $X$ and is defined by:

(Def. 9) $L$ where $O>O_{2}=\left\{x: \overline{\overline{x O_{2}}} \in \overline{\overline{x O}} \wedge x \in L\right\}$.

Let us consider $X, L, O, n$. The functor $L$ where $O=n$ yielding a list of $X$ is defined as follows:

(Def. 10) $L$ where $O=n=\{x: \overline{\overline{x O}}=n \wedge x \in L\}$.

The functor $L$ where $O \leq n$ yielding a list of $X$ is defined by:

(Def. 11) $L$ where $O \leq n=\{x: \overline{\overline{x O}} \subseteq n \wedge x \in L\}$.

The functor $L$ where $O \geq n$ yielding a list of $X$ is defined as follows:

(Def. 12) $L$ where $O \geq n=\{x: n \subseteq \overline{\overline{x O}} \wedge x \in L\}$.

The functor $L$ where $O<n$ yields a list of $X$ and is defined as follows:

(Def. 13) $L$ where $O<n=\{x: \overline{\overline{x O}} \in n \wedge x \in L\}$.

The functor $L$ where $O>n$ yields a list of $X$ and is defined by:

(Def. 14) $L$ where $O>n=\{x: n \in \overline{\overline{x O}} \wedge x \in L\}$.

One can prove the following propositions:

(3) $x \in L$ where $O$ iff $x \in L$ and $x O \neq \emptyset$.

(4) $L$ where $O \subseteq L$.

(5) If $L \subseteq \operatorname{dom} O$, then $L$ where $O=L$.

(6) If $n \neq 0$ and $L_{1} \subseteq L_{2}$, then $L_{1}$ where $O \geq n \subseteq L_{2}$ where $O$.

(7) $L$ where $O \geq 1=L$ where $O$. 
(8) If $L_{1} \subseteq L_{2}$, then $L_{1}$ where $O>n \subseteq L_{2}$ where $O$.

(9) $L$ where $O>0=L$ where $O$.

(10) If $n \neq 0$ and $L_{1} \subseteq L_{2}$, then $L_{1}$ where $O=n \subseteq L_{2}$ where $O$.

(11) $L$ where $O \geq n+1=L$ where $O>n$.

(12) $L$ where $O \leq n=L$ where $O<n+1$.

(13) If $n \leq m$ and $L_{1} \subseteq L_{2}$ and $O_{1} \subseteq O_{2}$, then $L_{1}$ where $O_{1} \geq m \subseteq$ $L_{2}$ where $\mathrm{O}_{2} \geq n$.

(14) If $n \leq m$ and $L_{1} \subseteq L_{2}$ and $O_{1} \subseteq O_{2}$, then $L_{1}$ where $O_{1}>m \subseteq$ $L_{2}$ where $\mathrm{O}_{2}>n$.

(15) If $n \leq m$ and $L_{1} \subseteq L_{2}$ and $O_{1} \subseteq O_{2}$, then $L_{1}$ where $O_{2} \leq n \subseteq$ $L_{2}$ where $O_{1} \leq m$.

(16) If $n \leq m$ and $L_{1} \subseteq L_{2}$ and $O_{1} \subseteq O_{2}$, then $L_{1}$ where $O_{2}<n \subseteq$ $L_{2}$ where $O_{1}<m$.

(17) If $O_{1} \subseteq O_{2}$ and $L_{1} \subseteq L_{2}$ and $O \subseteq O_{3}$, then $L_{1}$ where $O \geq O_{2} \subseteq$ $L_{2}$ where $O_{3} \geq O_{1}$.

(18) If $O_{1} \subseteq O_{2}$ and $L_{1} \subseteq L_{2}$ and $O \subseteq O_{3}$, then $L_{1}$ where $O>O_{2} \subseteq$ $L_{2}$ where $O_{3}>O_{1}$.

(19) If $O_{1} \subseteq O_{2}$ and $L_{1} \subseteq L_{2}$ and $O \subseteq O_{3}$, then $L_{1}$ where $O_{3} \leq O_{1} \subseteq$ $L_{2}$ where $\mathrm{O} \leq \mathrm{O}_{2}$.

(20) If $O_{1} \subseteq O_{2}$ and $L_{1} \subseteq L_{2}$ and $O \subseteq O_{3}$, then $L_{1}$ where $O_{3}<O_{1} \subseteq$ $L_{2}$ where $O<O_{2}$.

(21) $L$ where $O>O_{1} \subseteq L$ where $O$.

(22) If $O_{1} \subseteq O_{2}$ and $L_{1} \subseteq L_{2}$, then $L_{1}$ where $O_{1} \subseteq L_{2}$ where $O_{2}$.

(23) $a \in L \mid O$ iff there exists $b$ such that $a \in b O$ and $b \in L$.

Let us consider $X, A, B$. We introduce $A$ and $B$ as a synonym of $A \cap B$. We introduce $A$ or $B$ as a synonym of $A \cup B$. We introduce $A$ butnot $B$ as a synonym of $A \backslash B$.

Let us consider $X, A, B$. Then $A$ and $B$ is a list of $X$. Then $A$ or $B$ is a list of $X$. Then $A$ butnot $B$ is a list of $X$.

We now state several propositions:

(24) If $L_{1} \neq \emptyset$ and $L_{2} \neq \emptyset$, then $\left(L_{1}\right.$ or $\left.L_{2}\right) \& O=\left(L_{1} \& O\right)$ and $\left(L_{2} \& O\right)$.

(25) If $L_{1} \subseteq L_{2}$ and $O_{1} \subseteq O_{2}$, then $L_{1}\left|O_{1} \subseteq L_{2}\right| O_{2}$.

(26) If $O_{1} \subseteq O_{2}$, then $L \& O_{1} \subseteq L \& O_{2}$.

(27) $L \&\left(O_{1}\right.$ and $\left.O_{2}\right)=\left(L \& O_{1}\right)$ and $\left(L \& O_{2}\right)$.

(28) If $L_{1} \neq \emptyset$ and $L_{1} \subseteq L_{2}$, then $L_{2} \& O \subseteq L_{1} \& O$. 


\section{OpERATIONS}

One can prove the following two propositions:

(29) For all operations $O_{1}, O_{2}$ of $X$ such that for every $x$ holds $x O_{1}=x O_{2}$ holds $O_{1}=O_{2}$.

(30) For all operations $O_{1}, O_{2}$ of $X$ such that for every $L$ holds $L\left|O_{1}=L\right| O_{2}$ holds $O_{1}=O_{2}$.

The functor not $O$ yielding an operation of $X$ is defined as follows:

(Def. 15) For every $L$ holds $L \mid$ not $O=\bigcup\{(x O=\emptyset \rightarrow\{x\}, \emptyset): x \in L\}$.

Let us consider $X$ and let $O_{1}, O_{2}$ be operations of $X$. We introduce $O_{1}$ and $O_{2}$ as a synonym of $O_{1} \cap O_{2}$. We introduce $O_{1}$ or $O_{2}$ as a synonym of $O_{1} \cup O_{2}$. We introduce $O_{1}$ butnot $O_{2}$ as a synonym of $O_{1} \backslash O_{2}$. We introduce $O_{1} \mid O_{2}$ as a synonym of $\mathrm{O}_{1} \cdot \mathrm{O}_{2}$.

Let us consider $X$ and let $O_{1}, O_{2}$ be operations of $X$. Then $O_{1}$ and $O_{2}$ is an operation of $X$ and it can be characterized by the condition:

(Def. 16) For every $L$ holds $L \mid\left(O_{1}\right.$ and $\left.O_{2}\right)=\bigcup\left\{\left(x O_{1}\right)\right.$ and $\left.\left(x O_{2}\right): x \in L\right\}$.

Then $O_{1}$ or $O_{2}$ is an operation of $X$ and it can be characterized by the condition:

(Def. 17) For every $L$ holds $L \mid\left(O_{1}\right.$ or $\left.O_{2}\right)=\bigcup\left\{\left(x O_{1}\right)\right.$ or $\left.\left(x O_{2}\right): x \in L\right\}$.

Then $O_{1}$ butnot $O_{2}$ is an operation of $X$ and it can be characterized by the condition:

(Def. 18) For every $L$ holds $L \mid\left(O_{1}\right.$ butnot $\left.O_{2}\right)=\bigcup\left\{\left(x O_{1}\right) \operatorname{butnot}\left(x O_{2}\right): x \in L\right\}$.

Then $O_{1} \mid O_{2}$ is an operation of $X$ and it can be characterized by the condition:

(Def. 19) For every $L$ holds $L\left|\left(O_{1} \mid O_{2}\right)=L\right| O_{1} \mid O_{2}$.

The functor $O_{1} \& O_{2}$ yielding an operation of $X$ is defined as follows:

(Def. 20) For every $L$ holds $L \mid\left(O_{1} \& O_{2}\right)=\bigcup\left\{\left(x O_{1}\right) \& O_{2}: x \in L\right\}$.

We now state a number of propositions:

(31) $x\left(O_{1}\right.$ and $\left.O_{2}\right)=\left(x O_{1}\right)$ and $\left(x O_{2}\right)$.

(32) $x\left(O_{1}\right.$ or $\left.O_{2}\right)=\left(x O_{1}\right)$ or $\left(x O_{2}\right)$.

(33) $x\left(O_{1}\right.$ butnot $\left.O_{2}\right)=\left(x O_{1}\right) \operatorname{butnot}\left(x O_{2}\right)$.

(34) $x\left(O_{1} \mid O_{2}\right)=\left(x O_{1}\right) \mid O_{2}$.

(35) $x\left(O_{1} \& O_{2}\right)=\left(x O_{1}\right) \& O_{2}$.

(36) $z, s \in \operatorname{not} O$ iff $z=s$ and $z \in X$ and $z \notin \operatorname{dom} O$.

(37) $\operatorname{not} O=\operatorname{id}_{X \backslash \operatorname{dom} O}$.

(38) $\operatorname{dom} \operatorname{not} \operatorname{not} O=\operatorname{dom} O$.

(39) $L$ where not not $O=L$ where $O$.

(40) $L$ where $O=0=L$ where not $O$.

(41) $\operatorname{not} \operatorname{not} \operatorname{not} O=\operatorname{not} O$.

(42) $\operatorname{not} O_{1}$ or not $O_{2} \subseteq \operatorname{not}\left(O_{1}\right.$ and $\left.O_{2}\right)$. 
(43) $\operatorname{not}\left(O_{1}\right.$ or $\left.O_{2}\right)=\operatorname{not} O_{1}$ and not $O_{2}$.

(44) If $\operatorname{dom} O_{1}=X$ and $\operatorname{dom} O_{2}=X$, then $\left(O_{1}\right.$ or $\left.O_{2}\right) \& O=$ $\left(O_{1} \& O\right)$ and $\left(O_{2} \& O\right)$.

Let us consider $X, O$. We say that $O$ is filtering if and only if:

(Def. 21) $O \subseteq \operatorname{id}_{X}$.

Next we state the proposition

(45) $O$ is filtering iff $O=\operatorname{id}_{\operatorname{dom} O}$.

Let us consider $X, O$. Note that not $O$ is filtering.

Let us consider $X$. Note that there exists an operation of $X$ which is filtering.

In the sequel $F_{1}, F_{2}$ denote filtering operations of $X$.

Let us consider $X, F, O$. One can check the following observations:

* $F$ and $O$ is filtering,

* $O$ and $F$ is filtering, and

* $F$ butnot $O$ is filtering.

Let us consider $X, F_{1}, F_{2}$. One can verify that $F_{1}$ or $F_{2}$ is filtering.

(46) If $z \in x F$, then $z=x$.

(47) $L \mid F=L$ where $F$.

(48) $\operatorname{not} \operatorname{not} F=F$.

(49) $\operatorname{not}\left(F_{1}\right.$ and $\left.F_{2}\right)=\operatorname{not} F_{1}$ or not $F_{2}$.

(50) $\operatorname{dom}(O$ or not $O)=X$.

(51) $F$ or not $F=\operatorname{id}_{X}$.

(52) $O$ and not $O=\emptyset$.

(53) $\left(O_{1}\right.$ or $\left.O_{2}\right)$ and not $O_{1} \subseteq O_{2}$.

\section{Rough Queries}

Let $A$ be a finite sequence and let $a$ be a set. The functor \#occurrences $(a, A)$ yielding a natural number is defined as follows:

(Def. 22) \#occurrences $(a, A)=\overline{\overline{\{i: i \in \operatorname{dom} A \wedge a \in A(i)\}}}$.

We now state two propositions:

(54) For every finite sequence $A$ and for every set $a$ holds \#occurrences $(a, A) \leq$ len $A$.

(55) For every finite sequence $A$ and for every set $a$ holds $A \neq \emptyset$ and \#occurrences $(a, A)=\operatorname{len} A$ iff $a \in \bigcap \operatorname{rng} A$.

The functor max\# $A$ yielding a natural number is defined as follows:

(Def. 23) For every set $a$ holds \#occurrences $(a, A) \leq \max \# A$ and for every $n$ such that for every set $a$ holds \# occurrences $(a, A) \leq n$ holds $\max \# A \leq n$. 
(56) For every finite sequence $A$ holds $\max \# A \leq \operatorname{len} A$.

(57) For every finite sequence $A$ and for every set $a$ such that \#occurrences $(a, A)=\operatorname{len} A$ holds max\# $A=\operatorname{len} A$.

Let us consider $X$, let $A$ be a finite sequence of elements of $2^{X}$, and let $n$ be a natural number. The functor rough $n(A)$ yields a list of $X$ and is defined as follows:

(Def. 24) $\operatorname{rough} n(A)=\{x: n \leq \# \operatorname{occurrences}(x, A)\}$ if $X \neq \emptyset$.

Let $m$ be a natural number. The functor rough $n-m(A)$ yields a list of $X$ and is defined by:

(Def. 25) $\operatorname{rough} n-m(A)=\{x: n \leq \# \operatorname{occurrences}(x, A) \wedge \# \operatorname{occurrences}(x, A) \leq$ $m\}$ if $X \neq \emptyset$.

Let us consider $X$ and let $A$ be a finite sequence of elements of $2^{X}$. The functor $\operatorname{rough}(A)$ yielding a list of $X$ is defined by:

(Def. 26) $\operatorname{rough}(A)=$ rough max\# $A(A)$.

Next we state several propositions:

(58) For every finite sequence $A$ of elements of $2^{X}$ holds rough $n$ - len $A(A)=$ rough $n(A)$.

(59) For every finite sequence $A$ of elements of $2^{X}$ such that $n \leq m$ holds $\operatorname{rough} m(A) \subseteq \operatorname{rough} n(A)$.

(60) Let $A$ be a finite sequence of elements of $2^{X}$ and $n_{1}, n_{2}, m_{1}, m_{2}$ be natural numbers. If $n_{1} \leq m_{1}$ and $n_{2} \leq m_{2}$, then rough $m_{1}-n_{2}(A) \subseteq$ rough $n_{1}-m_{2}(A)$.

(61) For every finite sequence $A$ of elements of $2^{X}$ holds rough $n-m(A) \subseteq$ rough $n(A)$.

(62) For every finite sequence $A$ of elements of $2^{X}$ such that $A \neq \emptyset$ holds rough len $A(A)=\bigcap \operatorname{rng} A$.

(63) For every finite sequence $A$ of elements of $2^{X}$ holds rough $1(A)=\bigcup A$.

(64) For all lists $L_{1}, L_{2}$ of $X$ holds rough $2\left(\left\langle L_{1}, L_{2}\right\rangle\right)=L_{1}$ and $L_{2}$.

(65) For all lists $L_{1}, L_{2}$ of $X$ holds rough $1\left(\left\langle L_{1}, L_{2}\right\rangle\right)=L_{1}$ or $L_{2}$.

\section{Constructor Database}

We introduce constructor databases which are extensions of 1-sorted structures and are systems

$\langle$ a carrier, constructors, a ref-operation 〉,

where the carrier is a set, the constructors constitute a list of the carrier, and the ref-operation is a relation between the carrier and the constructors.

Let $X$ be a 1-sorted structure. A list of $X$ is a list of the carrier of $X$. An operation of $X$ is an operation of the carrier of $X$. 
Let us consider $X$, let $S$ be a subset of $X$, and let $R$ be a relation between $X$ and $S$. The functor ${ }^{\circledR} R$ yields a binary relation on $X$ and is defined by:

(Def. 27) ${ }^{@} R=R$.

Let $X$ be a constructor database and let $a$ be an element of $X$. The functor $a$ ref yielding a list of $X$ is defined as follows:

(Def. 28) $a$ ref $=a^{\circledR}$ the ref-operation of $X$.

The functor $a$ occur yields a list of $X$ and is defined as follows:

(Def. 29) $\quad a$ occur $=a\left({ }^{\circledR} \text { the ref-operation of } X\right)^{\smile}$.

The following proposition is true

(66) For every constructor database $X$ and for all elements $x, y$ of $X$ holds $x \in y$ ref iff $y \in x$ occur.

Let $X$ be a constructor database. We say that $X$ is ref-finite if and only if:

(Def. 30) For every element $x$ of $X$ holds $x$ ref is finite.

One can verify that every constructor database which is finite is also reffinite.

Let us note that there exists a constructor database which is finite and non empty.

Let $X$ be a ref-finite constructor database and let $x$ be an element of $X$. Observe that $x$ ref is finite.

Let $X$ be a constructor database and let $A$ be a finite sequence of elements of the constructors of $X$. The functor atleast $(A)$ yielding a list of $X$ is defined by:

(Def. 31) atleast $(A)=\{x \in X: \operatorname{rng} A \subseteq x$ ref $\}$ if the carrier of $X \neq \emptyset$.

The functor atmost $(A)$ yielding a list of $X$ is defined as follows:

(Def. 32) $\operatorname{atmost}(A)=\{x \in X: x \operatorname{ref} \subseteq \operatorname{rng} A\}$ if the carrier of $X \neq \emptyset$.

The functor exactly $(A)$ yields a list of $X$ and is defined by:

(Def. 33) $\operatorname{exactly}(A)=\{x \in X: x$ ref $=\operatorname{rng} A\}$ if the carrier of $X \neq \emptyset$.

Let $n$ be a natural number. The functor atleast minus $n(A)$ yields a list of $X$ and is defined by:

(Def. 34) atleast minus $n(A)=\{x \in X: \overline{\overline{\operatorname{rng} A \backslash x \text { ref }}} \leq n\}$ if the carrier of $X \neq \emptyset$.

Let $X$ be a ref-finite constructor database, let $A$ be a finite sequence of elements of the constructors of $X$, and let $n$ be a natural number. The functor atmost plus $n(A)$ yields a list of $X$ and is defined by:

(Def. 35) atmost plus $n(A)=\{x \in X: \overline{\overline{x \text { ref } \backslash \operatorname{rng} A}} \leq n\}$ if the carrier of $X \neq \emptyset$.

Let $m$ be a natural number. The functor exactly plus $n$ minus $m(A)$ yielding a list of $X$ is defined by: 
(Def. 36) exactly plus $n \operatorname{minus} m(A)=\{x \in X: \overline{\overline{x \operatorname{ref} \backslash \operatorname{rng} A}} \leq n \wedge$ $\overline{\overline{\operatorname{rng} A \backslash x \text { ref }}} \leq m\}$ if the carrier of $X \neq \emptyset$.

In the sequel $X$ denotes a constructor database, $x$ denotes an element of $X$, $B$ denotes a finite sequence of elements of the constructors of $Y$, and $y$ denotes an element of $Y$.

The following propositions are true:

(67) atleast minus $0(A)=\operatorname{atleast}(A)$.

(68) atmost plus $0(B)=\operatorname{atmost}(B)$.

(69) exactly plus 0 minus $0(B)=\operatorname{exactly}(B)$.

(70) If $n \leq m$, then atleast minus $n(A) \subseteq$ atleast minus $m(A)$.

(71) If $n \leq m$, then atmost plus $n(B) \subseteq$ atmost plus $m(B)$.

(72) For all natural numbers $n_{1}, n_{2}, m_{1}, m_{2}$ such that $n_{1} \leq m_{1}$ and $n_{2} \leq m_{2}$ holds exactly plus $n_{1}$ minus $n_{2}(B) \subseteq$ exactly plus $m_{1}$ minus $m_{2}(B)$.

(73) atleast $(A) \subseteq$ atleast minus $n(A)$.

(74) $\operatorname{atmost}(B) \subseteq$ atmost plus $n(B)$.

(75) exactly $(B) \subseteq$ exactly plus $n$ minus $m(B)$.

(76) $\operatorname{exactly}(A)=\operatorname{atleast}(A)$ and $\operatorname{atmost}(A)$.

(77) exactly plus $n$ minus $m(B)=$ atleast minus $m(B)$ and atmost plus $n(B)$.

(78) If $A \neq \emptyset$, then atleast $(A)=\bigcap\{x$ occur : $x \in \operatorname{rng} A\}$.

(79) For all elements $c_{1}, c_{2}$ of $X$ such that $A=\left\langle c_{1}, c_{2}\right\rangle$ holds atleast $(A)=$ $c_{1}$ occur and $c_{2}$ occur .

\section{REFERENCES}

[1] Grzegorz Bancerek. Cardinal numbers. Formalized Mathematics, 1(2):377-382, 1990.

[2] Grzegorz Bancerek. The fundamental properties of natural numbers. Formalized Mathematics, 1(1):41-46, 1990.

[3] Grzegorz Bancerek. The ordinal numbers. Formalized Mathematics, 1(1):91-96, 1990.

[4] Grzegorz Bancerek. Information retrieval and rendering with MML query. LNCS, 4108:266-279, 2006.

[5] Grzegorz Bancerek and Krzysztof Hryniewiecki. Segments of natural numbers and finite sequences. Formalized Mathematics, 1(1):107-114, 1990.

[6] Czesław Byliński. Functions and their basic properties. Formalized Mathematics, 1(1):5565, 1990.

[7] Czesław Byliński. Some basic properties of sets. Formalized Mathematics, 1(1):47-53, 1990.

[8] Agata Darmochwał. Finite sets. Formalized Mathematics, 1(1):165-167, 1990.

[9] Beata Padlewska. Families of sets. Formalized Mathematics, 1(1):147-152, 1990.

[10] Andrzej Trybulec. Binary operations applied to functions. Formalized Mathematics, 1(2):329-334, 1990.

[11] Wojciech A. Trybulec. Pigeon hole principle. Formalized Mathematics, 1(3):575-579, 1990.

[12] Zinaida Trybulec. Properties of subsets. Formalized Mathematics, 1(1):67-71, 1990.

[13] Edmund Woronowicz. Relations and their basic properties. Formalized Mathematics, 1(1):73-83, 1990.

[14] Edmund Woronowicz. Relations defined on sets. Formalized Mathematics, 1(1):181-186, 1990. 
[15] Bo Zhang, Hiroshi Yamazaki, and Yatsuka Nakamura. Set sequences and monotone class. Formalized Mathematics, 13(4):435-441, 2005.

Received December 18, 2011 